\title{
Aerosinusitis: Pathophysiology, Prophylaxis, and Management in Passengers and Aircrew
}

\author{
Erik K. Weitzel, K. Christopher McMains, \\ Suresh Rajapaksa, AND Peter-John Wormald
}

\begin{abstract}
Weitzel EK, McMains KC, Rajapaksa S, Wormald P-J. Aerosinusitis: pathophysiology, prophylaxis, and management in passengers and aircrew. Aviat Space Environ Med 2008; 79:50-3.

Patients presenting before flight with an upper respiratory infection are at risk for aerosinusitis. Prophylaxis of this condition consists of an oral decongestant before flight and nasal decongestant spray during the flight just prior to descent. Evaluation of the patient presenting with aerosinusitis consists of a careful physical exam with emphasis on diagnosing treatable nasal and sinus pathology. Categorization of the patient into the Weissman classification is important for determining prognostic factors for recovery. Management of this condition is based on the Weissman stage. Stage I or II lesions are generally treated conservatively with a 1-wk course of topical sprays, analgesics, a tapering course of steroids, and oral decongestants. Use of antibiotics is reserved for those cases initiated by bacterial sinusitis. Additionally, antihistamines are reserved for cases where allergies were the inciting cause. Stage III lesions are rarely seen in civilian air travelers due to the relatively low fluctuations in ambient air pressure. Aircrew that suffer Stage III aerosinusitis are at risk for recurrent sinus barotrauma that may require an expertly performed functional endoscopic sinus surgery to successfully manage it.

Keywords: sinus barotrauma, rhinologic barosinusitis.
\end{abstract}

\begin{abstract}
A PATIENT presenting with an upper respiratory tract infection in preparation for an airline flight is a frequent clinical scenario. Although there are a tremendous number of considerations in flight medicine (2), the most common flight-related disorders to be considered are barotrauma to the middle ear space and the paranasal sinuses.

Sinus barotrauma, or aerosinusitis, is $4-6$ times less common than middle ear barotrauma $(8,12,20)$. Since the sinuses are not equipped with an active pressure equalization system like the Eustachian tube, rhinologic pressure differences cannot be controlled and can cause devastating sequelae. Thus, sinus barotrauma tends to be more dramatic in presentation and the more difficult to manage of the two. This article focuses on the physics, clinical presentation, evaluation, and treatment of sinus barotrauma.
\end{abstract}

\section{Background}

Sinus barotrauma can occur during either ascent or descent. Although descent barotrauma or "squeeze" is more common by a ratio of at least 2:1 $(5,18)$, ascent barotrauma or "reverse squeeze" has more severe sequelae. Squeeze occurs due to a ball-valve effect caused by intranasal pathology. During ascent, atmospheric pressure decreases and gases within the sinuses expand, escaping through the sinus ostium. During descent, the ostium becomes obstructed by a ball-valve effect, prohibiting additional air from entering the sinus. This results in negative pressure within the sinus. The negative intrasinus pressure developed during a squeeze generally causes pain and mucosal edema, but in severe cases causes mucosal hemorrhage. Risk factors for squeeze include mucosal edema of tissues surrounding the sinus ostia, pus, thick mucin, extrasinus polyps, and tumors. It is currently not known if otherwise benign radiological anomalies such as septal deviation and concha bullosa place patients at increased risk for squeeze. However, when coupled with mucosal edema, these same space-occupying bony structures will tend to magnify the obstructive effects. Reverse squeeze can occur from the same activities that cause squeeze (15), but may also be caused by any phenomenon which forces air into the sinuses (e.g., mask ventilation). Reverse squeeze generally results from intrasinus pathology; however, tumors (20), skullbase bony dehiscences (21), and dehiscent cranial nerves $(7,17,18)$ have been implicated as well. During ascent, intrasinus pathology will act in a ball-valve fashion to obstruct the maxillary ostium and prevent air escape. Pressurized air will eventually dissect into surrounding tissue, finding a path of least resistance, leading to pathologic sequelae. The air that escapes the sinus by non-physiologic routes during a reverse squeeze can cause severe consequences, including subcutaneous or orbital emphysema, blindness, pneumocephalus, meningitis, and trigeminal nerve dysfunction (1).

From the Department of Surgery, Otolaryngology Head \& Neck Surgery, The Queen Elizabeth Hospital, Woodville, SA, Australia (E. K. Weitzel, S. Rajapaksa, P-J. Wormald) and the Department of Otolaryngology, University of Texas Health Science Center at San Antonio, San Antonio, TX (K. C. McMains).

This manuscript was received for review in September 2007. It was accepted for publication in October 2007.

Address reprint requests to: Maj. Erik Weitzel, M.D., MC, USAF, ENT Department, Queen Elizabeth Hospital, 28 Woodville Rd., Woodville, SA 5011, Australia; erik.weitzel@mac.com.

Reprint \& Copyright (C) by the Aerospace Medical Association, Alexandria, VA.

DOI: $10.3357 /$ ASEM.2203.2008 
Sinus barotrauma can be explained by Boyle's law. This law states that, at constant temperature, a volume of dry gas varies inversely with surrounding pressure $\left(\mathrm{V}=\mathrm{C}_{\mathrm{T}} / \mathrm{P}\right)$. This implies that an elastic chamber filled with air will expand as surrounding pressure decreases, as seen with increased altitude. This expansion occurs even more rapidly with the humidified or "wet" air that exists within the paranasal sinus cavities (20). As an example, at sea level, atmospheric pressure is $760 \mathrm{mmHg}$; at maximum decompression, the typical commercial airliner pressurizes to $560 \mathrm{mmHg}$, the equivalent of $8000 \mathrm{ft}$ of elevation $(11,22)$. By the ideal gas law $(\mathrm{PV}=\mathrm{nRT})$, dry air will expand/contract by more than one-third its original volume on a standard flight. Factoring in the humidified status of intrasinus air, this expansion becomes even more dramatic. Clinically, this means that a typical passenger will have to accommodate significant gas exchange during both ascent and descent within their sinuses during any commercial flight or expect to suffer some degree of barotrauma.

The rate of descent is also an important factor in the development of barotrauma. The faster the change in ambient pressure, the less time the sinus has to adjust before developing a critical pressure differential. Typical descent rates experienced by passengers on a civilian aircraft are $300-350 \mathrm{ft} \cdot \mathrm{min}^{-1}$, whereas military jet fighters expose their crew to descent rates in excess of 10,000 $\mathrm{ft} \cdot \mathrm{min}^{-1}$ (12). Negative intrasinus pressure of 100-150 $\mathrm{mmHg}$ creates mild barotrauma with mucosal edema or serous discharge. Severe cases of barotrauma with mucosal bleeding are noted when pressure differentials reach $260-300 \mathrm{mmHg}$ (23). Two authors have suggested that as extravascular blood accumulates, the mucosa tears from the periosteum and forms submucosal hematomas $(10,14)$. This has been confirmed by MRI (16) and plain radiograph (19). Since the maximal pressure differential sustained on a commercial flight is $200 \mathrm{mmHg}$, severe cases of sinus barotrauma are not reported with civilian air travel. However, with military flights, pressure differentials of $360 \mathrm{mmHg}$ (equivalent of $20,000 \mathrm{ft}$ elevation) can be experienced by the aircrew, surpassing the $260-\mathrm{mmHg}$ threshold to cause mucosal bleeding and thus lead to severe barotrauma.

\section{Clinical Presentation}

The incidence of sinus barotrauma is correlated to the physiologic demands placed upon the sinus cavities. Military pilot recruits and flight surgeons have the highest rates of significant barotrauma (1.5-4\%) during military aircraft simulations in hypobaric chambers $(10,19)$. The next most common group to experience aerosinusitis is patients exposed to much less aggressive dive rates in therapeutic hyperbaric chambers $(1.2 \%)$ (15). Severe sinus barotrauma is generally not reported in civilian air travel, although mild cases do occur. The frontal sinus is most commonly affected $(70-80 \%)$, followed by the maxillary sinus (19-29\%) $(6,10,19,20)$. There is about a $10 \%$ incidence of overlap between frontal and maxillary sinus barotrauma (6). The ethmoids and sphenoid sinuses have rarely been implicated in this condition $(10,18)$.

Aerosinusitis diagnosis requires the coincident occurrence of a sinus cavity pressure differential and sinus related discomfort (20). The pain is usually localized over the frontal or maxillary sinuses, but may also be retroorbital or in the region of maxillary dentition (19). Numerous case series in military pilots confirm the predominant symptom of frontal pain $(97 \%)$, followed by malar pain (27\%) and bloody rhinorrhea (13-58\%). Frontal pain is also the most common symptom in civilian barotrauma; however, bloody discharge has rarely been reported in this demographic $(18,22)$. This gives clinical support to the physical principles mentioned earlier, suggesting that the typical airline passenger suffering from sinus barotrauma is not exposed to pressure differentials large enough to cause mucosal hemorrhage. The Weissman classification system correlates the clinical aspects of sinus barotrauma to radiographic examination (Table I).

\section{Prophylaxis and Treatment}

Work-up for sinus barotrauma includes a thorough history to determine the preflight risk factors. Radiographs are then obtained to stage the patient according to Weissman classification (19). The first step in prophylaxis is identifying the at-risk individual. The major identifiable preflight risk factors for civilian aerosinusitis include active upper respiratory infection and allergic rhinitis $(5,14,20)$. In addition, the patient history should be queried for repeated episodes of sinus barotrauma, which is generally restricted to military aircrew, but conceivably exists in civilian patients with nasal polyps and ball-valving soft tissue structures (recurrent sinus barotrauma).

Prophylactic treatment for at-risk individuals should include an oral decongestant before flight and nasal decongestant spray during the flight just prior to descent (20). Although there have been no rigorously controlled trials of prophylactic medical regimens with sinus barotrauma, there is ample clinical experience to suggest the benefit of topical and oral vasoconstrictors. An important subpopulation of air travelers to be considered is the pregnant patient with rhinitis. This patient creates a complex problem since rhinitis occurs commonly during pregnancy, but standard medical treatments represent possible risks to the fetus. For a pregnant patient with severe rhinitis, the risks and

TABLE I. WEISSMAN CLASSIFICATION.

\begin{tabular}{|c|c|c|c|}
\hline & Symptoms & Plain Films & Pathology \\
\hline Class I & $\begin{array}{l}\text { Transient } \\
\text { discomfort }\end{array}$ & Normal & Slight swelling \\
\hline Class II & $<24 \mathrm{~h}$ pain & $\begin{array}{l}\text { Mucosal } \\
\text { thickening }\end{array}$ & $\begin{array}{l}\text { Serosanguinous } \\
\text { rhinorrhea }\end{array}$ \\
\hline Class III & $\begin{array}{l}\text { Severe pain } \\
\text { (squeeze } \\
\text { only) }\end{array}$ & Obliterated sinus & $\begin{array}{l}\text { Hematoma/mucosal } \\
\text { avulsion }\end{array}$ \\
\hline
\end{tabular}

Garges modification to Weissman's classification of sinus barotrauma (7). 
benefits in light of the reasons for travel should be discussed with the patient. Second and third trimester patients can be safely given small doses of pseudoephedrine and oxymetazoline in line with recommendations for typical air travelers. However, these same medications may confer unnecessary risks during the first trimester. During this 3-mo period, a few days course of topical nasal steroid may be useful for effective preflight decongestion (9).

In the military setting, in-flight management of acute sinus barotrauma typically involves ascending to an asymptomatic elevation and administering a nasal decongestant or performing a Valsalva to equalize the sinuses. After resolution of the pain, a slowed descent usually prevents further symptoms $(10,19)$. However, on a commercial airline flight, unless the person experiencing barotrauma is the pilot, the symptomatic individual will have to endure the symptoms until they exit the aircraft.

The treatment standard for postflight acute barotrauma is nasal decongestion (20). A typical regimen includes a 1-wk course of topical sprays, analgesics, a tapering course of steroids, and oral decongestants (19). Use of antibiotics is reserved for those cases initiated by bacterial sinusitis $(10,19,20)$. Additionally, antihistamines are reserved for cases where allergies were the inciting cause (10). In more severe cases mandating immediate intervention, Kraus recommends topical vasoconstriction followed by a Proetz displacement procedure and in-fracture of the middle turbinates (10). Resistant cases may require a maxillary or frontal trephination (10).

Patients experiencing acute aerosinusitis should be restricted from flight until all evidence of the barotrauma and its inciting risk factors have resolved. Minimum activity restriction includes a 1-wk period restricted from flying, swimming, or diving (19). Radiographs are repeated at $1 \mathrm{wk}$, and if evidence of mucosal thickening persists, the treating physician will decide on the duration of flight restrictions that may extend to $6 \mathrm{wk}$ (19). However, if the radiographs and symptoms have cleared or only show clinically irrelevant submucosal hematomas, the patient may be cleared for flight (1). At $6 \mathrm{wk}$, a repeat exam and CT scan are performed to reassess recovery. Since baseline mucosal thickening can exist in asymptomatic individuals $(4,12)$, this should not be the sole criterion for continued restriction from flight. After a severe episode of sinus barotrauma, pilots may be required to pass an altitude exposure in a hypobaric chamber prior to returning to duty (19) with rates of descent dependent on the aircraft flown.

Surgical management of Weissman Class III acute barosinusitis is rarely indicated; however, functional endoscopic sinus surgery (FESS) can be entertained if the patient has persistent pain with abnormal radiographs despite a reasonable period of medical management. In the military context, there are special circumstances which necessitate early surgery when personnel are of extraordinary operational value who must re-engage in activities likely to expose them to pressure changes prior to a normal period of recovery (18).

\section{Recurrent Sinus Barotrauma (RSB)}

Patients with RSB have repeated attacks of sinus barotrauma caused by returning to flying before adequate healing. Between attacks, patients are asymptomatic and have normal radiographs (1). It is hypothesized that the process is initiated with a case of severe barotrauma that causes avulsion of mucosa from the periosteum (Weissman Class III). During the healing process, substantial mucosal thickening occurs, increasing the potential for ostial narrowing (14). Other inciting and contributing causes that must be ruled out include chronic rhinosinusitis, polypoid sinonasal disease, or anatomic abnormalities, which may include concha bullosa, septal deviation, Haller cells, paradoxically curved uncinate/ middle turbinate, or other incidental findings that can lead to osteomeatal complex obstruction when coupled with mucosal edema $(13,14)$.

RSB is difficult to manage with conservative measures (3). First-line therapy includes antihistamines and nasal steroids. If chronic sinusitis is suspected, a 1-mo course of antibiotics is given. After resolution of symptoms, altitude chamber testing for pilots will determine if return to flight duty is possible (3). When medical therapy fails and CT scans suggest an anatomic problem, surgical intervention should be considered in an attempt to avoid permanent loss of flight status.

Recurrent sinus barotrauma is more commonly seen in military aircrew than in the civilian sector and was historically considered a career-ending diagnosis for pilots. Three studies show that traditional, non-FESS techniques have at best a $60-69 \%$ chance of returning a pilot to flight status (22). The application of FESS to RSB was first described by Bolger, Parsons, and Matson (3), who compared results in 10 cases of FESS to 19 cases treated by classic methods. The FESS group had a 100\% return to flight duty, whereas $36.8 \%$ of the control group returned to flight duty. The group concluded that once a diagnosis of RSB unresponsive to medical treatment is made, a CT scan followed by "full house FESS" (surgical opening of all sinuses), $6 \mathrm{wk}$ recovery, and a successful hypobaric dive will allow aviators to return to duty (13).

Two studies followed on to confirm this finding. O'Reilly first showed that FESS could return $95 \%$ of RSB patients to duty (12). Next, long-term follow up of a 54-aviator cohort by Parsons et al. showed that with FESS, $98 \%$ of aviators successfully returned to flight duty (14). Importantly, all previous traditional sinus surgery failures in this cohort were salvageable with FESS. Closer inspection of the series shows that a number of patients failed their initial FESS management due to frontal recess and lateral nasal wall synechiae. Revision FESS was able to salvage these cases as well (14). Given these data, RSB is a highly treatable condition and should no longer be considered a career-ending diagnosis for an aviator.

\section{Conclusions}

Although there are few scientific studies on this subject, the pathophysiology and physics of this condition 
are very clearly understood. Application of these principles to clinical medicine appears to have good outcomes. However, this conclusion must be tempered by the lack of data to prove this. A controlled assessment of aerosinusitis and its medical management is clearly needed.

A preflight physical examination including nasal endoscopy should identify any obvious intranasal obstructive edema or lesions blocking airflow into and out of the sinuses. Treatment of otherwise innocuous anatomical anomalies such as septal deviation and concha bullosa is currently not indicated to prevent barotrauma, but prospective cohort analysis to comprehend risk factors for aerosinusitis remain lacking. Mucosal edema caused by an upper respiratory infection or allergic rhinitis is the most commonly associated risk factor for civilian passengers. Edema is managed prophy lactically with an oral decongestant prior to flight and a topical decongestant just prior to descent. If barosinusitis does develop during ascent or descent during a civilian flight, it is highly unlikely that there would be any long-term sequelae since pressure differentials are comparatively low and resulting injury will be Weissman Class I or II. The pregnant air passenger with rhinitis currently represents a dilemma for the physician attempting to offer recommendations. Without further research, current literature supports standard medical management during second and third trimesters and nasal steroid alone during the first trimester (9).

After the acute event, management of sinus barotrauma relies on oral and topical decongestants ( \pm steroids). Periodic re-examination is performed until all evidence of the inciting event has resolved. In those instances that surgery is considered, a sinus surgeon experienced in the management of the frontal recess should be consulted to target either a specific anatomic abnormality or perform complete spheno-ethmoidectomies with maxillary antrostomies and frontal recess clearance (3).

Pilots and aircrew that have suffered through barotrauma require a higher level of rehabilitation, as their work environment puts them at high risk of recurrence. Special consideration must be given to the military aircrew as the pressure differentials experienced during military flight can lead to higher classes of barotrauma and risk of recurrent sinus barotrauma. Management of these patients should be in conjunction with an aerospace medicine doctor who is familiar with the physiologic stresses anticipated in their aircraft.

\section{REFERENCES}

1. Becker GD, Parell GJ. Barotrauma of the ears and sinuses after scuba diving. Eur Arch Otorhinolaryngol 2001; 258:159-63.

2. Bettes TN, McKenas DK. Medical advice for commercial air travelers. Am Fam Physician 1999; 60:801-10.

3. Bolger WE, Parsons DS, Matson RE. Functional endoscopic sinus surgery in aviators with recurrent sinus barotrauma. Aviat Space Environ Med 1990; 61:148-56.

4. Brandt MT. Oral and maxillofacial aspects of diving medicine. Mil Med 2004; 169:137-41.

5. Cheshire WP. Headache and facial pain in scuba divers. Curr Pain Headache Rep 2004; 8:315-20.

6. Dickson ED, King PF. Results of treatment of otitic and sinus barotrauma. J Aviat Med 1956; 27:92-9.

7. Garges LM. Maxillary sinus barotrauma-case report and review. Aviat Space Environ Med 1985; 56:796-802.

8. Hanna HH. Aeromedical aspects of otolaryngology. Aviat Space Environ Med 1979; 50:280-3.

9. Incaudo GA, Takach TP. The diagnosis and treatment of allergic rhinitis during pregnancy and lactation. Immunol Allergy Clin North Am 2006; 26:137-54.

10. Kraus RN. Treatment of sinus barotrauma. Ann Otol Rhinol Laryngol 1959; 68:80-9.

11. Lewis ST. Barotrauma in United States Air Force accidentslincidents. Aerosp Med 1973; 44:1059-61.

12. O'Reilly BJ, Lupa H, Mcrae A. The application of endoscopic sinus surgery to the treatment of recurrent sinus barotrauma. Clin Otolaryngol Allied Sci 1996; 21:528-32.

13. O'Reilly BJ, McRae A, Lupa H. The role of functional endoscopic sinus surgery in the management of recurrent sinus barotrauma. Aviat Space Environ Med 1995; 66:876-9.

14. Parsons DS, Chambers DW, Boyd EM. Long-term follow-up of aviators after functional endoscopic sinus surgery for sinus barotrauma. Aviat Space Environ Med 1997; 68:1029-34.

15. Plafki C, Peters P, Almeling M, Welslau W, Busch R. Complications and side effects of hyperbaric oxygen therapy. Aviat Space Environ Med 2000; 71:119-24.

16. Segev $Y$, Landsberg R, Fliss DM. MR imaging appearance of frontal sinus barotrauma. AJNR Am J Neuroradiol 2003; 24:346-7.

17. Sharma N, De M, Pracy P. Recurrent facial paraesthesia secondary to maxillary antral cyst and dehiscent infraorbital canal: case report. J Laryngol Otol 2007; 121:e6.

18. Singletary EM, Reilly JF Jr. Acute frontal sinus barotrauma. Am J Emerg Med 1990; 8:329-31.

19. Smith JP, Furry DE. Aeromedical considerations in the management of paranasal sinus barotrauma. Aerosp Med 1972; 43:1031-3.

20. Stewart TW, Jr. Common otolaryngologic problems of flying. Am Fam Physician 1979; 19:113-9.

21. Tryggvason G, Briem B, Guomundsson O, Einarsdottir H. Sphenoid sinus barotrauma with intracranial air in sella turcica after diving. Acta Radiol 2006; 47:872-4.

22. Wolf CR. Aerotitis in air travel. Calif Med 1972; 117:10-2.

23. Yanagawa Y, Okada Y, Ishida K, Fukuda H, Hirata F, Fujita K. Magnetic resonance imaging of the paranasal sinuses in divers. Aviat Space Environ Med 1998; 69:50-2. 